\title{
Ischemic stroke and acute pemphigus - Two partners in crime
}

\author{
Maria Liliana Gealapu', Ana Maria Enascuta ${ }^{1}$, Cristina Georgiana Croitoru', \\ Alexandra Liliana Popa', Cristina Burtea', Daniel Naconecinii', \\ Dan Iulian Cuciureanu, ${ }^{1,2}$ \\ ${ }^{1}$ Emergency Clinic Hospital "Prof. Dr. N. Oblu", lasi, Romania \\ 2University of Medicine and Pharmacy "Gr. T. Popa", lasi, Romania
}

\begin{abstract}
Introduction. Bullous dermatoses are a group of skin-specific autoimmune diseases with systemic involvement that have a molecular basis related to the development of $\mathrm{IgG}$ autoantibodies targeted against desmosome proteins. The purpose of this report consists in marking the association between an acute cerebrovascular event and an active phase blistering dermatosis, at a patient with no other known risk factors.

Case presentation. Female patient, 46 years old, diagnosed with pemphigus vulgaris one year before, was admitted to the emergency room with left hemiparesis. The general physical examination shows multiple fragile blisters with serocitrin content and well-defined painful skin erosion, localized on the limbs, thorax and abdomen. The neurological examination revealed a conscious, cooperative, right-handed patient with left proportional flaccid hemiparesis, and homonimous hemianopya. Native cranio-cerebral computed-tomography described acute infarction in the right sylvian arterial territory. Extensive paraclinical tests did not reveal an evident pathogenesis of acute ischemic stroke.

Conclusions. Patients with bullous dermatosis have a two-folded increased risk of stroke, particularly ischemic stroke and this risk remains high even after controlling modifiable cardiovascular risk factors. The neurologist should consider implementing primary ischemic stroke prevention in patients diagnosed with bullous dermatosis.
\end{abstract}

Keywords: stroke, pemphigus vulgaris, bullous dermatosis

\section{INTRODUCTION}

Autoimmune bullous skin diseases are characterized by an immune response against structural proteins of the skin and mucous membrane. The diagnosis is based on the clinical, histological and immunological aspects. In the medical literature there are described two main bullous skin diseases: the pemphigus group and the sub-epidermal autoimmune blistering diseases (1).

Pemphigus is characterized by the loss of intercellular adhesion at epidermal and epithelial mucous membrane level, induced by auto antibodies against desmogleins 1 (Dsg 1) and 3 (Dsg 3) from the desmosomes structure. By breaking the adhesion intercellular structures, intraepidermic cleavage occurs seen in histopathological examination. These microscopic changes develop and turn into clinical lesions in the form of cutaneous flaccid blisters and erosions on the mucous membrane level (1). Patients also develop antibodies against desmosomal proteins, such as desmocollins and plakins and non-desmosomal proteins, such as nicotinic acetylcholine receptor, pemphaxin, tyroperoxidase and annexins. These data indicate that pemphigus is a complex disease, initiated by at least three classes of autoantibodies that explain the acantholysis in the disease (2). Both direct and indirect immuno-fluorescence reveal a typical aspect with an intercellular binding of immunoglobulin $\mathrm{G}$ (Ig G) and complement C3 in the epidermis and epithelium (1).

The most frequent disease of subepidermal blistering group is bullous pemphigoid, characterized by auto antibodies against two components of hem- 
idesmosomes, BP180 and BP230. Clinically, the patients suffering from bullous pemphigoid present tense blisters, usually only on the skin level, and histological examination shows subepidermal blisters. Direct immunofluorescence reveals linear deposition of $\operatorname{IgG}$ at the dermoepidermal junction (1).

The incidence of pemphigus vulgaris is 0.68 per 100,000 person years and the incidence of bullous pemphigoid is 4.28 per 100,000 person years (3).

\section{CASE REPORT}

A 46-year-old, right handed woman has been brought to the emergency room for confusion, proportional motor deficit on the left side of the body and facial asymmetry. These symptoms developed within 24 hours prior to admission. Her family history was insignificant. From her pathological background, she was diagnosed with pemphigus vulgaris one year ago and since then even though she underwent immunosuppressive treatment with Azathioprine and corticosteroid she presented several relapses. Clinical examination revealed an obese patient $(\mathrm{BMI}=32)$ with normal respiratory and cardio-vascular functions. When skin was examined, it presented multiple flaccid blisters filled with clear fluid that arised from an erythematous base and also painful and large erosions, localized on the limbs, thorax and abdomen (figure 1). When the neurological examination was performed at admission, it showed an oriented patient, with no fever and no stiff neck but with proportional left hemiparesis $(\mathrm{MRC}=0 / 5$, hypotonia, severely diminished osteotendinous reflex on the left side, left Babinsky sign), left facial central palsy and left homonymous hemianopia. The NIHSS score at admission was 13.

Differential diagnosis of specific cumulous of neurological deficits included complicated post-ictal Todd hemiparesis, intracerebral abscess, intracerebral tumour and acute cerebrovascular disease . However, the acute onset of a focal deficit is most suggestive for an acute cerebrovascular cause. In accordance with the international and national guidelines, facing the probability of acute stroke, a native computer tomography of the head was performed. This investigation revealed a hypodensity situated in the right internal capsule, suggestive for

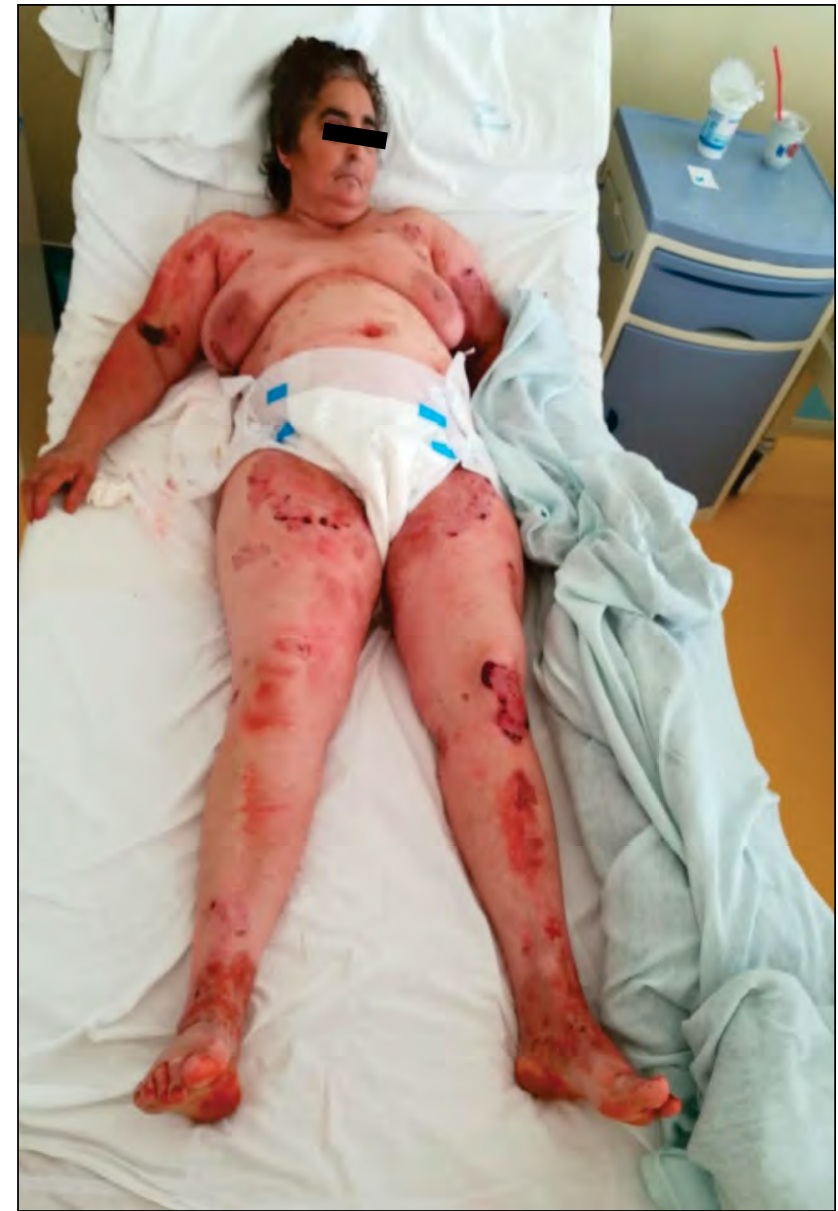

FIGURE 1. The multiple blisters that easily rupture, leaving behind painful sloughing eroded areas of skin on the limb and thorax and abdomen

an ischemic stroke in the profound territory of the right middle cerebral artery (figure 2). In order to to initially asses the patient, usual biochemical and haematological blood tests showed were performed, which revealed inflammatory syndrome (elevated C-reactive protein- $40 \mathrm{mg} / \mathrm{L}$ ), slight hyponatremia and haemoconcentration, the last two being suggestive for severe dehydration. Included in the etiological assessment, a number of additional paraclinical examinations were performed. Firstly, a neck vessels Doppler ultrasound was performed which revealed mild atherosclerosis of both carotid bifurcations. Secondly, the patient underwent a thorough cardiological examination including a transthoracic echocardiography which was negative for thrombus or severe atrial dilation. Given the important skin lesions on the thorax, no EKG Holter was able to be performed during hospitalization. In order to discover a possible procoagulant state due to hereditary or aquired thrombophilia, specific blood tests were performed (Factor V Lei- 
den, MTHFR mutation, PAI hyperexpression, protein $\mathrm{C}$ deficiency, protein $\mathrm{S}$ deficiency, antithrombin-III deficiency and antiphospholipid antibodys). However, all these tests were neggative.

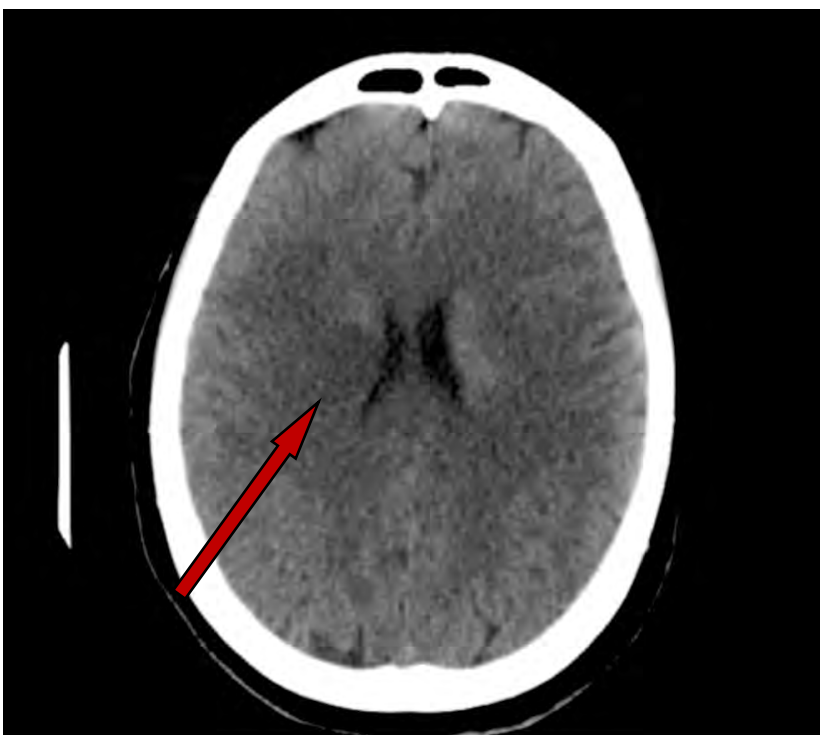

FIGURE 2. The native computer tomography of the head shows a hypodensity situated in the caudate nucleus/right internal capsule

When the etiopathology of an acute ischemic stroke in a relative young patient with scarce cardiovascular risk factors was discussed, several ideas emerged. Atherothrombotic mechanism is less probable due to lack of spontaneous hyperdense imaging in the right sylvian artery. The embolic arterio-arterial mechanism is possible because of the presence of atherosclerotic plaques, but without unstable plaque at the cervical Doppler ultrasound. The hemodynamic mechanism may be correlated with severe dehydration, but excluded due to the lack of low blood pressure at admission and characteristic localization of stroke (junctional). Embolic cardio-arterial mechanism was excluded because of lack of dyskinesias of ventricular walland intracardiac thrombus at transthoracic echocardiography. A less common cause of ischemic stroke but probable in a young patient is a procoagulant state due to hereditary or acquired thrombophilia. This could be ruled out due to the negativity of specific blood tests mentioned before.

Unfortunately, there is lack of information in the medical literature concerning the complex management of pacients which present an acute ischemic stroke and also are diagnosed with a bullous dermatosis prior to onset of motor deficit. Consequent- ly, an interdisciplinary approach was manadatory and the terapeutical guidelines for acute ischemic stroke were associated with topical and systemic treatment of the cutaneous lesions, in accordance with the dermatologic recommendations. To be more specific, the patient received aspirin and statin treatment for the secundary prevention of ischemic stroke and also genearal mesures were taken. For the prevention of immediate complications, especially pulmonary embolism and deep vein thrombosislow mollecular hepharine therapy was initiated with Nadroparinin accordance with the patient's weight $(0,4 \mathrm{ml}$ twice daily). For the management of the pemphigus vulgaris, the patient followed systemic therapy with prednison and azathioprine (intravenous pulses of methylprednisolone as initial treatment in severe disease followed by $1 \mathrm{mg} / \mathrm{kg} /$ day oral prednison and adjuvant immunosuppresant-Azathioprine $100 \mathrm{mg}$ /day) and local therapy for the skin lesions (gently clean blister with antimicrobial solution, taking care not to rupture, pierce the base of the blister with a sterile needle, with the bevel facing up, gently apply pressure with sterile gause swabs to facilitate drainage and absorbtion, after fluid has drained, gently apply again with an antimicrobial solution).

The clinical evolution was slightly favorable. The patient was discharged in the twelve day of hospitalization with a NIHSS score of 12 . The skin lesions showed signs of healling. She was strongly reccomended to undergo a minimum fourty-eight hours EKG HOLTER.

\section{DISCUSSION}

In the literature the prevalence of bullous dermatosis in the general population is 3.4 per 100000 inhabitants. There is cited an increased frequency of associations between this autoimmune skin disorders and several neurological diseases, among which the most frequent are neurocognitive disorders $(20 \%)$, ischemic stroke $(15 \%)$, parkinsonian syndromes $(9 \%)$, and other neurological diseases such as multiple sclerosis, lateral amiotrophic sclerosis, siringomyelia and epilepsy (5\%) (4).

Association with neurological diseases could be due to cross-reactivity between skin and neural antigens. The Dsg1, from the desmosomes structure of patients with pemphigus vulgaris, was found to 
be expressed in the corpus callosum of the mouse brain and was localized around the plasma membrane regions of oligodendrocytes. The desmoglein $1-\gamma$ expression is not restricted to the skin, as it is also expressed in the brain, skeletal muscle, and liver, among other tissues (5). At the patients with bullous pemphigoid, BP180 is strongly expressed in the cortex and hippocampus, the neuronal isoforms of BP230 are widely expressed in the human central and peripheral nervous system. The mechanism behind this association has been suggested to be an auto inflammatory reaction against BP180 or the neuronal isoform of BP230 in the human brain (6).

Increased risk of stroke in patients with bullous dermatosis can be explained by several possible mechanisms. The first mechanism implies the chronic inflammation with acute exacerbation during the skin relapses. Multiple proinflammatory cytokines, including interleukins (IL) such as IL-1, IL-2, IL-4, IL-5, IL-6, IL-8, IL-10, tumor necrosis factor $\alpha$ and interferon $\gamma$ are increased in the serum of patients with bullous dermatosis. Moreover, elevated serum levels of soluble E-selectin and vascular endothelial growth factor in bullous dermatosis further support the activation of endothelial inflammatory and immune reactions.

There is increasing evidence that the pathogenesis and progression of atherosclerosis, plaque rupture, thrombosis, and stroke involve inflammatory processes and endothelial dysfunction (7). The chronic inflammation and hypercoagulable state associated with bullous dermatosis can exacerbate pre-existing atheroma and promote thrombus formation. These processes predispose the patient to thrombotic events, particularly ischemic stroke (7).

Another possible explanation for the high risk of stroke in patients with bullous pemphigoid is a hypercoagulation state related to anti-phospholipid antibodies (aPLs). A recent study shows that aPLs were detected at fifty percent of patients with autoimmune blistering disease (7). This mechanism could not be ruled out because of the negativity of the serological tests since many studies have shown that the level of antiphospholipid antibodies can be controlled after three months of immunosuppressive treatment, the patient being on an immunosuppressive treatment with Azathioprine for approximately one year (8).
Regarding the cause of acute ischemic stroke in the clinical case presented, it is most likely that it is secondary to a cumulous of factors such severe dehydration which promotes a procoagulant state and atherosclerotic plaque exacerbated by chronic and acute systemic inflammation.

The high thrombotic tendencies found in patient with bullous dermatosis such as bullous pemphigoid, particularly evident during the acute phase of the disease suggest thromboembolism prophylaxis with antithrombotic drugs. The type of organs involved varied and included the brain. To be more specific, a case series revealed that thromboembolism occurred in $35 \%$ patients with autoimmune blistering disease, including bullous dermatosis (9). Therefore, the administration of Nadroparin in a prophylactic dose was highly justified in the presented clinical case not only because the patient was bed-bound with a severe motor deficit but also because patients with bullous dermatosis associate a high risk of venous thrombotic events.

For the prevention of vascular events we administrated to patient high dose corticotherapy. One of the common complications of high dose corticosteroid therapy is the increasing of cardiovascular risk (hypertension, myocardial infarction). However in the literature review no specific corelation was found between the increase risk of cerebrovascular events and high dose corticotherapy (10).

The poor prognosis is set since the first year after the neurological disorder onset, not only because the the relapse complications, superinfection with various organisms of the denudated, fever, but olso by increasing the arterial and venous vascular factor risk.

\section{CONCLUSIONS}

Cerebrovascular events are the second cause of neurological disease in pemphigus pacients, after neurocognitive disorders. Patients with bullous dermatosis have a two-folded increased risk of stroke, particularly ischemic stroke and this risk remains high even after controlling modifiable cardiovascular risk factors.

Patients with bullous pemphigoid with acute stroke are more likely to experience post stroke respiratory and urinary tract infections and subsequent poor outcome because glucocorticoid related im- 
munosuppression. Therefore, they should not overlook the increased risk of stroke after the onset of bullous disease.

The existance of a chronic proinflammatory background, associated with an increased inflammatory status during relapse periods resulting in endothelial dysfunction and progression of preexisting atheromatosis, the increasing risk of thrombosis in acute phase and a probable hypercoagulable state are the most presumably mechanisms in the pathogenesis of vascular events found in pacients diagnosted with bullous cutaneous disease, in the absence of other risk factors.
Taking everything into account, firstly dermatologists but actually all specialities should not overlook the incresead risk of stroke after the onset of bullous diseases. In response, the neurologist should consider implementing primary ischemic stroke prevention in patients diagnosed with bullous dermatosis.

Further studies that include clinical markers of bullous dermatosis severity, serum level of proinflammatory cytokines and of antiphospholipid antibodies are required in order to clarify the underlying pathomechanisms between pemphigus vulgaris and stroke.
Conflict of interest: none declared Financial support: none declared

\section{REFERENCES}

1. Baican C, Baican A, Rogojan L, Ciuce D, Samasca G, Macovei V, Perta A, Maier N, Sitaru C. The sensitivity of clinical, histologic and immunologic findings for the diagnosis of autoimmune bullous disease. DermatoVenerol. 2011; 56:11-19.

2. Pan M, Liu X, Zheng J. The pathogenic role of autoantibodies in pemphigus vulgaris;Clin Exp Dermatol. 2011; 36(7):703-7.

3. Langan SM, Smeeth L, Hubbard R, Fleming KM, Smith CJP. Bullous pemphigoid and pemphigus vulgaris - incidence and mortality in the UK: population based cohort study J West, clinician scientist3 BMJ 2008; 337:a180.

4. Cordel N, Chosidow O, Hellot MF, Delaporte E, Lok C, Vaillant L, Bernard P, D'Incan M, Roujeau JC, Joly P. The French Study Group of Bullous Diseases Neurological Disorders in Patients with Bullous Pemphigoid, Dermatology. 2007; 215(3):187-91.

5. Kridin K, Zelber-Sagi S, Comaneshter D, Cohen AD. Association Between Pemphigus and Neurologic Diseases, JAMADermatology, JAMA Dermatol. 2018:154(3):281-285.

6. Casas-de-la-Asunción E, Ruano-Ruiz J, Rodríguez-Martín AM, Vélez García-Nieto A, Moreno-Giménez JC. Association Between Bullous

Pemphigoid and Neurologic Diseases: A Case-Control Study; Actas Dermo-Sifiliográficas (English Edition), 2014; 105: 860-865.

7. Yang YW, Chen YH, Xirasagar S, Lin HC. Increased Risk of Stroke in Patients With Bullous Pemphigoid A Population-Based Follow-Up Stud, Stroke. 2011;42:319-323.

8. Jakubíková M, Pitha J, Latta J, Ehler E, Schutzner J. Myasthenia gravis, Castleman disease, pemphigus, and anti-phospholipid syndrome. Muscle and Nerve. 2013;47(3): 447-451.

9. Cugno M, Marzano AV, Bucciarelli P, Balice $Y$, Cianchini G, Quaglino P, Calzavara Pinton P, Caproni M, Alaibac M, De Simone C, Patrizi A, Cozzani E, Papini M, Tedeschi A, Berti E, Rosendaal FR, INVENTEP Study Group. Increased risk of venous thromboembolism in patients with bullous pemphigoid. The INVENTEP (INcidence of VENous ThromboEmbolism in bullous Pemphigoid) study; Thromb Haemost. 2016 ;115(1):193-9.

10. Ng MKC, Celermajer DS. Glucocorticoid treatment and cardiovascular disease; Heart 2004;90:829-830. 